\title{
Dacriocistorrinostomía endoscópica. Resultados en 172 pacientes
} Endoscopic dacryocystorhinostomy. Results in 172 patients

\author{
Eva Pablos-Buitrón ${ }^{1 *}$, Alejandro Martínez-Morán², Javier Lage-Fernández ${ }^{3}$, Patricia Cerdeira-Pena ${ }^{4}$ y \\ Vivian Pérez-Varela ${ }^{4}$ \\ ${ }^{1}$ Servicio de Otorrinolaringología, Hospital Universitario de Ferrol, Ferrol; ${ }^{2}$ Servicio de Otorrinolaringología, Hospital Universitario de A Coruña, A \\ Coruña; ${ }^{3}$ Servicio de Otorrinolaringología, Hospital La Rosaleda, Santiago de Compostela; ${ }^{4}$ Servicio de Oftalmología, Hospital Universitario de A \\ Coruña, A Coruña. España
}

\section{Resumen}

Introducción: La dacriocistorrinostomía es la técnica de elección para el tratamiento de la obstrucción de la vía lagrimal baja y de la dacriocistitis de repetición. A pesar de que la cirugía abierta tiene los mayores porcentajes de éxito, hoy en día las técnicas endoscópicas ofrecen ventajas en cuanto a duración de la cirugía, sencillez de la técnica, reproducibilidad, ausencia de cicatriz y disminución de las molestias para el paciente, con una alta tasa de éxito. Actualmente la técnica elegida en el Hospital Universitario de A Coruña es la dacriocistorrinostomía endoscópica. Objetivo: Se han recogido los datos de las cirugías realizadas durante 3 años con el objetivo de analizar los resultados comparándolos con los descritos en la literatura. Método: Se recogieron los resultados de 172 pacientes con un total de 220 dacriocistorrinostomías endoscópicas entre enero de 2014 y marzo de 2017. Resultados: El éxito según el test de permeabilidad fue del 83.5\% y solo el 12,7\% necesitaron una reintervención. Conclusiones: La dacriocistorrinostomía endoscópica es actualmente la técnica preferida por oftalmólogos y otorrinolaringólogos para el tratamiento de la obstrucción crónica de la vía lagrimal. Sus tasas de éxito son altas y ofrece múltiples ventajas sobre la técnica abierta.

Palabras clave: Obstrucción del conducto lacrimal. Endoscópica. Dacriocistorrinostomía. Ep ífora. Dacriocistitis.

\begin{abstract}
Introduction: Dacryocystorhinostomy is the technique of choice for the treatment of lower lacrimal duct obstruction and recurrent dacryocystitis. Although open surgery has the highest success rates, nowadays endoscopic techniques offer advantages in terms of duration of surgery, simplicity of the technique, reproducibility, absence of scarring, and reduction of discomfort for the patient, with a high success rate. Currently, endoscopic dacryocystorhinostomy is the chosen technique in the Hospital Universitario de A Coruña. Objective: The data of the surgeries performed for 3 years have been collected with the objective of analyzing the results comparing them with those described in the literature. Method: We analyzed the results of 172 patients with a total of 220 endoscopic dacryocystorhinostomies performed between January 2014 and March 2017. Results: According to the patency test, the success rate was $83.5 \%$, and only $12.7 \%$ required reoperation. Conclusions: Endoscopic dacryocystorhinostomy is currently the preferred technique for ophthalmologists and otorhinolaryngologists for the treatment of chronic lacrimal obstruction. Its success rates are high, and it offers multiple advantages over the open technique.
\end{abstract}

Key words: Lacrimal duct obstruction. Endoscopic. Dacryocystorhinostomy. Epiphora. Dacryocystitis. 


\section{Introducción}

La dacriocistorrinostomía (DCR) es la cirugía de elección para el tratamiento de la epífora de origen obstructivo de la vía lagrimal baja, así como en la dacriocistitis de repetición ${ }^{1,2}$, restableciendo el drenaje lagrimal hacia las fosas nasales.

Está descrito que, en la mayoría de los casos, la obstrucción de la vía lagrimal se debe a una alteración en el drenaje del saco lacrimal, por lo que dichos casos de epífora o infección del saco pueden ser resueltos mediante $\mathrm{DCR}^{3}$.

En nuestro hospital, el diagnóstico lo realiza el servicio de oftalmología en la sección de vías lacrimales mediante el test de lavado con cánula, y desde hace años la cirugía se realiza conjuntamente con el servicio de otorrinolaringología. Dicho test consiste en la canalización de uno de los puntos lagrimales y la instilación de solución salina fisiológica, con lo cual se observa, en caso de obstrucción sacal o postsacal, el reflujo por otro de los puntos. Son estos los pacientes en que esta cirugía es útil.

Aunque la técnica por abordaje externo es la que tiene mayores porcentajes de éxito ${ }^{4-6}$, en muchos hospitales se prefiere la técnica endoscópica porque presenta claras ventajas, que se fundamentan en la rapidez de los procedimientos, la recuperación de los pacientes, la reproducibilidad de la técnica y la ausencia de cicatriz. La DCR por abordaje externo supone un mayor tiempo quirúrgico, la necesidad de una incisión externa y mayor posibilidad de hemorragias ${ }^{7,8}$.

El objetivo de este estudio fue analizar los resultados de la DCR endoscópica en nuestro servicio, compararlos con los descritos en la literatura y describir las posibles complicaciones quirúrgicas.

\section{Método}

Se recogieron de manera retrospectiva los datos de los pacientes operados entre enero de 2014 y marzo de 2017, siendo un total de 172 pacientes. Los casos de cirugía bilateral se contabilizaron como dos procedimientos independientes, por lo que suman un total de 220 intervenciones, realizadas conjuntamente entre los servicios de oftalmología y de otorrinolaringología. Se incluyeron datos sobre la existencia de cirugía nasal previa, vasculitis, blefaritis o dacriocistitis, y también sobre las complicaciones, como epistaxis. El éxito anatómico se determinó teniendo en cuenta la comprobación de la permeabilidad y la ausencia de dacriocistitis, y el funcional o subjetivo con la mejoría de los síntomas que refieren los pacientes.

Los datos se analizaron usando el programa IBM SPSS Statistics 24.0, con la colaboración del departamento de estadística del hospital. Se realizó un análisis retrospectivo descriptivo de las variables recogidas en el estudio. Las variables cuantitativas se expresan como media \pm desviación típica, y las cualitativas como frecuencia y porcentaje.

La asociación entre variables cualitativas se estimó por medio de la prueba de ji al cuadrado o test de Fisher. Se analizó, mediante modelos de regresión logística, el impacto de las diferentes variables en la necesidad de una segunda intervención, ajustando por aquellas que resultaron significativas en el análisis univariante o por aquellas clínicamente relevantes.

\section{Técnica quirúrgica}

En la mayoría de los casos, los pacientes fueron operados con anestesia local y sedación en el quirófano regular y programado.

Se administraron gotas tópicas de anestésico doble sobre la conjuntiva y con el mismo anestésico se realizó de nuevo, para una comprobación final, el test de permeabilidad.

La fosa nasal se exploró previamente a la cirugía para descartar variaciones anatómicas que dificultasen el acceso a la pared lateral de la fosa, como desviaciones del tabique o conchas bullosas. En las cirugías se utilizaron endoscopios de $4 \mathrm{~mm}$ y $30^{\circ}$. Se introdujeron lentinas empapadas en anestésico doble y epinefrina al 1:1000 en el meato medio y sobre los cornetes inferior y medio.

Posteriormente se infiltró con escandicaína y epinefrina la mucosa que recubre la apófisis frontal del maxilar y el hueso lacrimal. Se esperó entre 5 y 10 minutos para conseguir el máximo efecto de la anestesia y de la vasoconstricción.

Con un bisturí falciforme se incidió sobre la mucosa para descubrir la apófisis frontal del hueso maxilar y se realizó un pequeño colgajo mucoso para favorecer la exposición.

Con dos pinzas tipo Smith-Kerrison de $2 \mathrm{~mm}$ y angulaciones de $90^{\circ}$ y $45^{\circ}$ se extrajeron varios fragmentos de la apófisis frontal del maxilar y del hueso lagrimal, y para visualizar la cara medial del saco.

Se realizó entonces el sondaje de la vía lagrimal a través de la sonda de Bowman, favoreciendo la protrusión de su pared e incidiendo el saco con el bisturí 
oftalmológico falciforme. La pared medial del saco se resecó con la técnica de sacabocados.

Se llevó a cabo el sondaje de la vía lagrimal con la sonda bicanalicular de silicona. Se utilizó en todos los pacientes, para evitar la cicatrización y el cierre de la osteotomía, mitomicina tópica al 0,4\% durante 5 minutos, con posterior lavado de la zona con solución salina.

Finalmente, se realizó un taponamiento hasta que el paciente fue dado de alta a su domicilio, con una serie de instrucciones que incluyen el lavado de la fosa nasal con solución salina a partir del día siguiente a la cirugía, colirio con antibiótico y corticoides, y analgesia.

En las reintervenciones se resecó la cicatriz previa, se realizó el sondaje y de nuevo se aplicó mitomicina $C$.

Se revisó a todos los pacientes a los 15 días del procedimiento en el servicio de otorrinolaringología. En esta consulta se trató de eliminar las posibles costras endonasales de la zona operada. La siguiente cita, tras comprobar la cicatrización favorable de la zona operada, fue a los 2 meses de la cirugía para la retirada de la sonda. Las revisiones sucesivas se realizaron en el servicio de oftalmología, donde se evaluó la permeabilidad de la vía lacrimal mediante la técnica de sondaje e irrigación, la curación de las infecciones en caso de dacriocistitis y la resolución de los síntomas de los pacientes.

\section{Resultados}

Se analizaron los datos de 172 pacientes, sumando un total de 220 intervenciones. El $75 \%$ eran mujeres y el $25 \%$ hombres, con una media de edad de 70 años (rango: 31-94 años). En cuanto a los antecedentes personales, el $92 \%$ eran no fumadores, el $39 \%$ tomaban antiagregantes y el 50\% tomaban antihipertensivos.

Un total de 74 (33.6\%) habían presentado episodios de dacriocistitis y $36(16.4 \%)$ de blefaritis. Solo 20 casos de los 200 fueron reintervenciones $(9.09 \%)$. En cuanto a la cirugía, el $70.9 \%$ fue unilateral y el $71.8 \%$ se realizó con anestesia local y sedación. El periodo de seguimiento medio fue de 14 meses (rango: 12-35 meses).

El éxito anatómico, según el test de permeabilidad al final del seguimiento, fue del $83.6 \%$. El éxito subjetivo, según la satisfacción de los pacientes en cuanto al cese total de los síntomas, fue del $77 \%$. El $12.7 \%$ de los casos requirió otra intervención.

Mediante modelos de regresión logística univariantes se objetiva que la dacriocistitis y la blefaritis son las únicas variables con efecto independiente para predecir la necesidad de una segunda intervención. Los pacientes con dacriocistitis previa presentan menor probabilidad de reintervención (odds ratio [OR]: 0.19; intervalo de confianza [IC]: 0.10-0.90). Por el contrario, los pacientes con blefaritis tienen seis veces más riesgo de necesitar una reintervención (OR: 6.03; IC: 2.55-14.27). Se encuentran en el límite de la significación el sexo $(p=0.089)$ y el tratamiento antiagregante $(p=0.069)$. El sexo masculino presenta menor riesgo de reintervención, y el hecho de recibir tratamiento antiagregante (OR: 0.15 ) disminuye la probabilidad de necesitar una nueva intervención. La edad no presenta un impacto estadísticamente significativo, aunque a mayor edad (OR: 0.99; IC: 0.96-1.02) se observa una tendencia a la disminución de la realización de una segunda intervención.

Ajustando por edad, sexo, dacriocistitis y blefaritis, se objetiva que la única variable con efecto independiente para predecir la necesidad de una nueva intervención es la blefaritis. Los pacientes con blefaritis (OR: 5.10; IC: 2.10-12.42) presentan cinco veces más riesgo de requerir una reintervención que los pacientes que no la sufren. Solo $4(1.8 \%)$ de los casos con dacriocistitis previas tuvieron otro episodio de infección tras la intervención.

En cuanto a las complicaciones, $4(1.8 \%)$ pacientes ingresaron a causa de epistaxis que precisó taponamiento anterior, y todos presentaron resolución de la epífora tras la cirugía.

\section{Discusión}

Aunque la DCR por abordaje externo es la técnica que presenta mayores tasas de éxito ${ }^{4-6,9}$, un $80-95 \% \%^{7,9-13}$, presenta claras desventajas respecto a la técnica endoscópica, como el mayor porcentaje de necesidad de anestesia general, el mayor tiempo quirúrgico ${ }^{5,14}$, la afectación del mecanismo valvular de la vía lagrimal y la cicatriz externa ${ }^{15}$. Con la técnica endoscópica, la cirugía puede realizarse en la mayoría de los casos con sedación y anestesia local, disminuyendo las molestias, y sin necesidad de ingreso, por lo que los pacientes de edad avanzada pueden optar por la intervención, que además presenta mínimas complicaciones; la más frecuente en nuestro grupo ha sido la epistaxis, en el $1.8 \%$ de los casos, que se resolvieron con un taponamiento nasal anterior.

Creemos necesaria la acción conjunta entre los servicios de otorrinolaringología y de oftalmología, ya que el diagnóstico lo establece el servicio de oftalmología 
y el abordaje quirúrgico endonasal lo realiza el servicio de otorrinolaringología.

Nuestra tasa de éxito es comparable a las reportadas en la literatura ${ }^{4,16,17}$, con una permeabilidad al final del seguimiento del $83.5 \%$ y una tasa de revisión de la cirugía del $12.7 \%$, lo que no suele significar un gran trastorno para el paciente incluso aunque tenga que pasar por una cirugía adicional.

Los resultados estadísticamente significativos que hemos encontrado son la dacriocistitis como factor protector y la blefaritis como factor de riesgo, en cuanto a necesidad de una segunda intervención.

Sobre el éxito de la cirugía con las diferentes técnicas, existe gran variabilidad de consideraciones en los artículos publicados. Los pacientes que acuden por infecciones de repetición suelen estar satisfechos si se resuelve la dacriocistitis crónica, aunque permanezca una leve epífora residual. Por ello, entendemos que la tasa de éxito debe medirse con el test de permeabilidad y la valoración subjetiva sobre el resultado de la cirugía según el paciente. En todo caso, consideramos que estamos ante un fracaso cuando planteamos al paciente la posibilidad de una nueva cirugía porque el test de lavado es negativo y el paciente se encuentra insatisfecho. Hay pacientes con buena permeabilidad de la vía lacrimal comprobada con el test de lavado que están insatisfechos; en algunos casos también puede haber patología del párpado asociada, y no se resolverá con una nueva DCR.

Wormald ${ }^{16}$ estudió la influencia de la talla de la osteotomía en el resultado de la cirugía y concluyó que con la exposición del saco lagrimal en su totalidad sus resultados eran comparables a los de la técnica abierta. Actualmente, entendemos que la osteotomía debe permitir el drenaje de la vía lagrimal lo más horizontal posible, por lo que debemos tratar de abrir el saco lagrimal en su totalidad, pero sobre todo en la región más superior.

Algunos autores refieren mejorar sus tasas de éxito si no resecan el colgajo mucoso y lo introducen en forma de solapa en el saco residual para favorecer la cicatrización ${ }^{15}$. Nosotros ya no realizamos esta maniobra porque, tal como se ha reportado en la literatura ${ }^{18}$, los resultados son comparables y la talla de dicho colgajo aumenta el tiempo quirúrgico.

Sobre la aplicación de mitomicina C, tampoco está clara su utilidad real. Parece mejorar el proceso de curación de la herida intranasal ${ }^{19}$, pero hay discordancia sobre su influencia en la tasa de éxito en las técnicas endoscópicas ${ }^{19,20}$, aunque sí que aporta mejorías significativas en las DCR externas y en la revisiones de DCR endoscópicas ${ }^{21}$. No hemos observado ninguna complicación por el uso de mitomicina.
Cuando empezamos con el abordaje endoscópico de la DCR se nos propuso inicialmente la posibilidad de realizar la osteotomía con láser, por las ventajas halladas por otros autores ${ }^{17,22,23}$. Durante unos meses utilizamos esta tecnología en nuestro servicio, pero finalmente había que ampliar la osteotomía con fresa o laminotomo, por lo que al final la abandonamos.

Pearlman et al. ${ }^{17}$ analizaron los resultados de la técnica endoscópica usando el láser YAG (itrio aluminio granate) y refieren la ventaja de menores complicaciones, con ningún caso de exposición de grasa orbitaria ${ }^{17}$.

Mann y Wormald ${ }^{24}$ estudiaron la variación del tamaño del ostium en el tiempo, y concluyeron que los cambios en el tamaño transcurren durante las primeras 4 semanas tras la cirugía; por lo tanto, creemos adecuado el tiempo de seguimiento de nuestro pacientes, con una media de 12 meses.

\section{Conclusiones}

Consideramos que la cirugía endoscópica para el tratamiento de la obstrucción de la vía lagrimal baja debe ser la técnica mayoritaria en nuestros hospitales. Se trata de un abordaje sencillo, seguro y rápido, con una alta tasa de éxito, lo que nos ha permitido aumentar su indicación en los pacientes de edad avanzada.

Como cirujanos, los otorrinolaringólogos y los oftalmólogos debemos colaborar para poder ofrecer este tipo de cirugía a los pacientes. Hoy en día, las tasas de éxito se acercan a las de la cirugía abierta convencional, con importantes beneficios sobre esta.

\section{Financiación}

No se recibió financiación para la elaboración de este trabajo.

\section{Conflicto de intereses}

Los autores declaran no tener ningún conflicto de intereses.

\section{Responsabilidades éticas}

Protección de personas y animales. Los autores declaran que para esta investigación no se han realizado experimentos en seres humanos ni en animales. 
Confidencialidad de los datos. Los autores declaran que han seguido los protocolos de su centro de trabajo sobre la publicación de datos de pacientes.

Derecho a la privacidad y consentimiento informado. Los autores han obtenido el consentimiento informado de los pacientes y/o sujetos referidos en el artículo. Este documento obra en poder del autor de correspondencia.

\section{Bibliografía}

1. Das SK, Sarkar P, Dan A, Boral K, Basak B, Banerjee SN. Endoscopic dacryocystorhinostomy: a study at IPGMER, Kolkata. Indian J Otolaryngol Head Neck Surg. 2013;65(Suppl 2):366-70.

2. Marcet MM, Kuk AK, Phelps PO. Evidence-based review of surgical practices in endoscopic endonasal dacryocystorhinostomy for primary acquired nasolacrimal duct obstruction and other new indications. Cur Opin Ophthalmol. 2014;25:443-8.

3. Avdagic E, Phelps PO. Nasolacrimal duct obstruction as an important cause of epiphora. Dis Mon. 2020;66:101043.

4. Hartikainen J, Antila J, Varpula M, Puukka P, Seppä H, Grénman R Prospective randomized comparison of endonasal endoscopic dacryocystorhinostomy and external dacryocystorhinostomy. Laryngoscope. 1998;108:1861-6.

5. Cokkeser $\mathrm{Y}$, Evereklioglu $\mathrm{C}, \mathrm{Er} \mathrm{H}$. Comparative external versus endoscopic dacryocystorhinostomy: results in 115 patients (130 eyes). Otolaryngol Head Neck Surg. 2000;123:488-91.

6. Tarbet KJ, Custer PL. External dacryocystorhinostomy. Ophthalmology. 1995;102:1065-70.

7. Huang J, Malek J, Chin D, Snidvongs K, Wilcsek G, Tumuluri K, et al Systematic review and meta-analysis on outcomes for endoscopic versus external dacryocystorhinostomy. Orbit. 2014;33:81-90.

8. Jawaheer L, MacEwen CJ, Anijeet D. Endonasal versus external dacryocystorhinostomy for nasolacrimal duct obstruction. Cochrane Database Syst Rev. 2017;(2):Cd007097.
9. Tarbet KJ, Custer PL. External dacryocystorhinostomy. Surgical success, patient satisfaction, and economic cost. Ophthalmology. 1995;102:1065-70.

10. Dolman PJ. Comparison of external dacryocystorhinostomy with nonlaser endonasal dacryocystorhinostomy. Ophthalmology. 2003;110:78-84.

11. Mekonnen W, Adamu Y. Outcome of external dacryocystorhinostomy in Ethiopian patients. Ethiop Med J. 2009;47:221-6.

12. Fayers $T$, Laverde $T$, Tay $E$, Olver JM. Lacrimal surgery success after external dacryocystorhinostomy: functional and anatomical results using strict outcome criteria. Ophthalmic Plast Reconstr Surg. 2009;25:472-5.

13. Warren JF, Seiff SR, Kavanagh MC. Long-term results of external dacryocystorhinostomy. Ophthalmic Surg Lasers Imaging. 2005;36:446-50.

14. Lee DW, Chai $\mathrm{CH}$, Loon SC. Primary external dacryocystorhinostomy versus primary endonasal dacryocystorhinostomy: a review. Clin Exp Ophthalmol. 2010;38:418-26.

15. Massegur Solench H, Trias Mis E, Ademà Alcover JM. Dacriocistorrinostomía endoscópica: técnica modificada. Acta Otorrinolaringol Esp. 2002;53:463-8.

16. Wormald PJ. Powered endoscopic dacryocystorhinostomy. Laryngoscope. 2002;112:69-72.

17. Pearlman SJ, Michalos $P$, Leib ML, Moazed KT. Translacrimal transnasal laser-assisted dacryocystorhinostomy. 1997;107:1362-5.

18. Kingdom TT, Barham HP, Durairaj VD. Long-term outcomes after endoscopic dacryocystorhinostomy without mucosal flap preservation. Laryngoscope. 2020;130:12-7.

19. Ragab SM, Elsherif HS, Shehata EM, Younes A, Gamea AM. Mitomycin C-enhanced revision endoscopic dacryocystorhinostomy: a prospective randomized controlled trial. Otolaryngol Head Neck Surg. 2012;147:93742.

20. Majhi S, Sharma A. Evaluation of role of mitomycin $C$ in the cases of nasolacrimal duct blockage undergoing endoscopic dacryocystorhinostomy. Indian J Otolaryngol Head Neck Surg. 2019;71(Suppl 3):1981-5.

21. Xue K, Mellington FE, Norris JH. Meta-analysis of the adjunctive use of mitomycin $\mathrm{C}$ in primary and revision, external and endonasal dacryocystorhinostomy. Orbit. 2014;33:239-44.

22. Metson R, Woog JJ, Puliafito CA. Endoscopic laser dacryocystorhinostomy. Laryngoscope. 1994;104:269-74.

23. Reifler DM. Results of endoscopie KTP laser-assisted dacryocystorhinostomy. Ophthalmic Plast Reconstr Surg. 1993;9:231-6.

24. Mann BS, Wormald PJ. Endoscopic assessment of the dacryocystorhinostomy ostium after endoscopic surgery. Laryngoscope. 2006;116:1172-4 